\title{
Qualidade de vida e satisfação em usuários de prótese total no estado do Amapá, Brasil
}

\author{
Quality of life and satisfaction in users of total prosthesis in the state of Amapá, Brazil
}

Calidad de vida y satisfacción en usuarios de prótesis total en el estado de Amapá, Brasil

Éber Coelho Paraguassu ${ }^{1 *}$, Karina da Silva Figueira1, Jamille dos Passos Lacerda1, Ulisses Gomes Guimarães Neto² e Carlos Eduardo Vieira Gomes ${ }^{3}$.

\section{RESUMO}

Objetivos: Os objetivos deste estudo foram avaliar e comparar o grau de satisfação e qualidade de vida de edêntulos usuários de prótese total na cidade de Macapá - Ap. Metodologia: Foram entrevistados 299 usuários de prótese total mucossuportada e 48 usuários de prótese total implantossuportada por meio de dois questionários, o Oral Health Impact Profile-14Br (OHIP-14Br) e a escala visual analógica (EVA) para satisfação com as próteses dentárias. Resultados: Ao realizar a avaliação da satisfação quanto ao tipo de prótese, os usuários de prótese implantossuportada estavam 100\% muito satisfeitos com a prótese superior e inferior e quanto à satisfação dos usuários de prótese mucossuportada, $90 \%$ dos usuários relataram estarem satisfeitos com a prótese superior, porém $56 \%$ demonstraram algum tipo de insatisfação com a prótese removível inferior. A avaliação do impacto da saúde bucal na qualidade de vida, em usuários de prótese implantossuportada, obteve um escore de 3,24 pontos na escala OHIP-14Br, enquanto os usuários de prótese mucossuportada tiveram um escore de 15,23 na escala OHIP-14Br.Conclusão: Conclui-se que usuários de prótese implantossuportada apresentaram significativamente melhor qualidade de vida e satisfação com suas próteses, quando comparados aos usuários de prótese total mucossuportada.

Palavras-Chave: Qualidade de vida, Prótese dentária, Saúde bucal.

\begin{abstract}
Objectives: The objectives of this study were to evaluate and compare the degree of satisfaction and quality of life of edentulous users of total prosthesis in the city of Macapá - Ap. Methods: A total of 299 users of a fullsize denture and 48 users of total denture implants were interviewed using two questionnaires, the Oral Health Impact Profile-14Br (OHIP-14Br) and the Visual Analogue Scale (VAS) for dental prostheses. Results: When assessing the satisfaction of the type of prosthesis, users of implanted prostheses were $100 \%$ very satisfied with the upper and lower prosthesis and regarding the satisfaction of users of a prosthesis with mucosa supported, $90 \%$ of the users reported being satisfied with the prosthesis $56 \%$ demonstrated some type of dissatisfaction with the lower removable prosthesis. The evaluation of the impact of oral health on the quality of life in users of implanted prostheses obtained a score of 3.24 points on the OHIP-14Br scale, while users of multisupported prostheses had a score of 15.23 on the OHIP-14Br scale. Conclusion: It was concluded that users of implant-supported prosthesis had significantly better quality of life and satisfaction with their prostheses when compared to users of a full-thickness prosthesis.
\end{abstract}

Key words: Quality of life, Dental prosthesis, Oral health.

${ }^{1}$ Goe/Uniavan Departamento de ciências odontológicas. Macapá- AP. *E-mail: paraguassu84@yahoo.com.br ${ }^{2}$ Faculdade de Macapá - Departamento de ciências odontológicas - Macapá- AP.

3Uninassau- Departamento de ciências odontológicas - Belém- PA. 


\section{RESUMEN}

Objetivos: Los objetivos de este estudio fueron evaluar y comparar el grado de satisfacción y calidad de vida de los usuarios edéntulos de prótesis totales en la ciudad de Macapá - Ap. Métodos: Se entrevistó a un total de 299 usuarios de una prótesis de tamaño completo y 48 usuarios de implantes de prótesis totales utilizando dos cuestionarios, el Perfil de impacto en la salud bucal-14Br (OHIP-14Br) y la Escala analógica visual (VAS) para prótesis dentales. Resultados: Al evaluar la satisfacción del tipo de prótesis, los usuarios de prótesis implantadas estaban $100 \%$ muy satisfechos con la prótesis superior e inferior y con respecto a la satisfacción de los usuarios de una prótesis con soporte mucoso, el $90 \%$ de los usuarios informó que estaba satisfecho con la prótesis. El $56 \%$ demostró algún tipo de insatisfacción con la prótesis removible inferior. La evaluación del impacto de la salud oral en la calidad de vida en los usuarios de prótesis implantadas obtuvo una puntuación de 3,24 puntos en la escala OHIP-14Br, mientras que los usuarios de prótesis con múltiples soportes obtuvieron una puntuación de 15,23 en la escala OHIP-14Br. Conclusión: se concluyó que los usuarios de prótesis con soporte de implantes tenían una calidad de vida y una satisfacción significativamente mejores con sus prótesis en comparación con los usuarios de una prótesis de espesor total.

Palabras clave: Calidad de vida, Prótesis dental, Salud bucal.

\section{INTRODUÇÃO}

Há um aumento do número de pessoas sem nenhum dente na boca, no Brasil, deficiência física que pode causar muitos problemas de saúde, como reabsorção óssea maxilomandibular, deficiências nutricionais motivadas pela inabilidade de mastigar alimentos sólidos, problemas psicológicos e de relacionamentos interpessoais, causando um impacto negativo na qualidade de vida (NICO LS, 2016).

Segundo o último censo da Pesquisa Nacional de Saúde (PNS), realizada pelo IBGE (Instituto Brasileiro de Geografia e Estatística) em 2013, em convênio com o Ministério da Saúde (MS), o Brasil tem 11\% da população desdentada total, o que corresponde a um montante de 16 milhões de pessoas. O número é mais prevalente em indivíduos com 60 anos ou mais, visto que $41,5 \%$ deles já perderam todos os dentes. Em $23 \%$ da população brasileira, há edentulismo em uma das duas arcadas e 33\% usam algum tipo de prótese dentária (NICO LS, 2016).

O Edentulismo total, que é a ausência de todos os dentes, é considerado uma deficiência física incapacitante pela Organização Mundial de Saúde (OMS) e, com a saúde física afetada, há um decréscimo na qualidade de vida dos desdentados totais (BRENNAN DS e SPENCER AJ, 2004). Uma solução satisfatória, para minimizar esta baixa qualidade de vida, é a reabilitação protética.

A pouca eficiência da função mastigatória, em desdentados usuários de próteses totais mucossuportadas (PTMS) maxilomandibular, tem sido observada na literatura (PRADO MMS, et al., 2004). A função mastigatória precária causa deglutição de pedaços muito grandes de alimentos ou em mudanças significativas da dieta, fazendo com que se evitem alimentos mais difíceis de serem mastigados e causando uma restrição alimentar (OLIVEIRA TRC, et al., 2007). Além disso, o esforço para mastigar alguns alimentos pode acarretar em constrangimentos às pessoas, o que pré-dispõe o aparecimento de distúrbios psicossociais que atingem negativamente a sua qualidade de vida e satisfação com as próteses (PARAGUASSU ÉC e CARDENAS AMC, 2019; SHEIHAM A, et al., 2001).

O fator de fixação e de estabilidade da prótese total implantossuportada (PTIS) minimiza o déficit mastigatório, proporcionando maior função mastigatória, conforto e segurança ao paciente, impactando assim positivamente sua qualidade de vida e satisfação com as próteses (RAES S, et al., 2017)

Diante deste cenário exposto e a fim de impulsionar novos conhecimentos nesta temática, foi levantado o problema, qual o grau de satisfação dos usuários de prótese total mucossuportada e prótese total implantossuportada e o quanto o uso contínuo destas próteses interfere na qualidade de vida dessas pessoas, no município de Macapá? 
Para responder a estas questões, os objetivos deste estudo foram avaliar e comparar o grau de satisfação e o impacto da saúde bucal na qualidade de vida de edêntulos totais usuários de prótese total mucossuportada e de prótese total implantossuportada, por meio de dois questionários, o Oral Health Impact Proifile-14Br (OHIP-14Br) e a escala visual analógica (EVA) para satisfação, no município de Macapá, estado do Amapá, Brasil.

Este estudo foi motivado devido a magnitude do número de desdentados totais e usuários de prótese total e o ineditismo deste tipo de estudo na região devido à falta de pesquisas relacionados a qualidade de vida e satisfação desta população no estado do Amapá e em toda região Amazônica.

\section{MATERIAIS E METODOS}

Trata-se de estudo descritivo transversal, com método comparativo e abordagem quantitativa de portadores de prótese total mucossuportada e implantossuportada e, depois, por meio de um questionário estruturado, obteve por análises estatísticas, o grau de satisfação com o uso de próteses bucais e a qualidade de vida dos dois grupos e fazer a comparação entre eles.

A pesquisa foi composta por pessoas desdentadas totais, portadoras de prótese total maxilomandibular do tipo mucossuportada ou implantossuportada, com até 10 anos de uso e moradoras da zona urbana do município de Macapá, que realizaram tratamento nas clínicas odontológicas privadas e do Centro de especialidades odontológicas (CEO-AP. Dessa forma, a população do estudo foi de 1.393 sujeitos, sendo 1.330 pacientes usuários de prótese total mucossuportada e 63 pacientes usuários de prótese total implantossuportada. Para a identificação do valor ideal da amostra, foi realizado um cálculo amostral, com a fórmula:

Figura 1 - Fórmula para cálculo amostral.

$$
n=\frac{N \cdot Z^{2} \cdot p \cdot(1-p)}{Z^{2} \cdot p \cdot(1-p)+e^{2} \cdot(N-1)}
$$

Fonte: Dados obtidos pelo programa SPPS STATISTICS 22.

Em que: $\mathrm{N}=1.393$ é o tamanho da população; $\mathrm{Z}$ - variável normal padronizada associada ao nível de confiança de $95 \% ; p=0,5$ é proporção verdadeira considerando margem máxima e $=0,05$ como erro amostral. A amostra necessária, para os pacientes usuários de prótese total mucossuportada, foi de 299, e a amostra necessária de pacientes usuários de prótese total implantossuportada foi de 48. Dos 299 usuários de prótese mucossuportada, 91 foram pacientes da rede de clínicas privadas e 208 do CEO-AP.

Foram incluídos na pesquisa desdentados totais, usuários de prótese total maxilomandibular com até 10 anos de uso do tipo mucossuportada e implantossuportada que morassem no município de Macapá. Excluíram-se da pesquisa qualquer outro tipo de situação protética, pacientes parcialmente dentados, pessoas que não morassem em Macapá ou que apresentassem aparente deficiência mental e ou que estivessem debilitadas fisicamente.

Para a coleta de dados, os pacientes foram contatados por telefone, e os interessados em participar da pesquisa receberam uma visita e responderam a dois questionários.

O primeiro questionário foi respondido, por meio de uma escala visual analógica (EVA), para avaliar a satisfação do paciente com as próteses. Foi composto de quatro perguntas sobre retenção, mastigação, satisfação e estética, que foram aplicadas para cada uma das próteses que o paciente utilizava.

A EVA, afere uma pontuação de 0 a 10 em cada um dos quesitos perguntados, chegando ao total de 40 pontos, sendo o resultado final dividido por quatro para se obter a nota de satisfação com a prótese. Resultados de 0 a 2,5 pontos correspondem a "muito insatisfeito"; de 2,75 a 5,0, "insatisfeito"; de 5,25 a 7,5 pontos, "satisfeito"; e de 7,75 a 10 pontos, "muito satisfeito" (JACOBOVITZ F, 2003). 
O segundo questionário é o OHIP-14br, na versão composta por 14 questões divididas em sete situações (JACOBOVITZ F, 2003): Limitações funcionais - questões 1 e 2; dor física - questões 3 e 4; desconforto psicológico - questões 5 e 6; incapacidade física - questões 7 e 8;

incapacidade psicológica - questões 9 e 10; incapacidade social - questões 11 e 12; invalidez questões 13 e 14.

Para calcular o impacto da saúde bucal na qualidade de vida dos pacientes, foi utilizado o método padrão de cálculo do OHIP-14, utilizando peso específico para cada questão. As seguintes pontuações serão atribuídas a cada resposta: Nunca $=0$; raramente $=1$; às vezes $=2$; frequentemente $=3$; sempre $=4$

Além disso, esse valor foi multiplicado pelo peso de cada pergunta como segue abaixo:

Quadro 1 - Valores de referência para a multiplicar com a pontuação obtida em cada pergunta na escala OHIP-14Br.

\begin{tabular}{|c|c|c|c|c|c|c|c|c|c|c|c|c|c|c|}
\hline Pergunta & 1 & 2 & 3 & 4 & 5 & 6 & 7 & 8 & 9 & 10 & 11 & 12 & 13 & 14 \\
\hline Peso & 0,51 & 0,49 & 0,34 & 0,66 & 0,45 & 0,55 & 0,52 & 0,48 & 0,6 & 0,4 & 0,62 & 0,38 & 0,59 & 0,41 \\
\hline
\end{tabular}

Fonte: Elaborado por Jacobovitz F, 2003.

Dessa forma, ao somar a pontuação final de todas as perguntas, os valores variaram entre 0 e 28 pontos. Quanto menor o valor na escala, melhor seria a qualidade de vida (Q.V). Valores de 0 a 9,33 representam boa Q.V, valores de 9,34 a 18,66 correspondem a uma razoável qualidade de vida e valores de 18,67 até 28 correspondem à má Q.V.

Este estudo atende aos critérios de ética em pesquisa com seres humanos, seguindo a resolução 466/2012 do conselho nacional de saúde, sendo aprovado pelo CEP/CONEP sob o parecer 2.451.731.

\section{RESULTADOS e DISCUSSÃO}

Os resultados das variáveis quanto à satisfação com as próteses implantossuportada e mucossportada estão descritas nas Tabelas 1 e 2.

Ao realizar a avaliação da satisfação dos pacientes quanto ao tipo de prótese, de acordo com a EVA, os pacientes com a prótese implantossuportada superior e inferior disseram estar muito satisfeitos, $100 \%(n=48)$. Quanto à satisfação do uso da prótese mucossuportada, 90\% $(n=269)$ dos pacientes relataram estar satisfeitos com a prótese superior, porém $56 \%$ demonstraram algum tipo de insatisfação com a prótese removível inferior (Tabela 1).

Tabela 1 - Satisfação da paciente com as próteses de acordo com o EVA. Macapá, AP, Brasil, 2018.

\begin{tabular}{lcccccccc}
\hline & \multicolumn{2}{c}{$\begin{array}{c}\text { Prótese implantossuportada } \\
\text { Prótese Superior }\end{array}$} & $\begin{array}{c}\text { Prótese Inferior } \\
\text { Classificação }\end{array}$ & \multicolumn{3}{c}{$\begin{array}{c}\text { Prótese mucossuportada } \\
\text { Prótese } \\
\text { Superior }\end{array}$} & $\begin{array}{c}\text { Prótese } \\
\text { Inferior }\end{array}$ \\
\cline { 2 - 9 } & $\mathrm{n}$ & $\%$ & $\mathrm{~N}$ & $\%$ & $\mathrm{n}$ & $\%$ & $\mathrm{~N}$ & $\%$ \\
\hline Muito satisfeito & 48 & 100 & 48 & 100 & 28 & 9 & 3 & 1 \\
Satisfeito & 0 & 0 & 0 & 0 & 269 & 90 & 112 & 38 \\
Insatisfeito & 0 & 0 & 0 & 0 & 2 & 1 & 168 & 56 \\
Muito insatisfeito & 0 & 0 & 0 & 0 & 0 & 0 & 16 & 5 \\
\hline
\end{tabular}

Fonte: Dados obtidos pelo autor no programa SPPS STATISTICS 22.

A satisfação quanto ao uso das próteses mostrou-se significativa entre os dois grupos. $100 \%$ dos usuários de prótese total superior e inferior implantossuportada estavam muito satisfeitos com a média de 9.39 pontos para a prótese superior e 9.47 pontos de média para a prótese inferior. 
Já os usuários de prótese mucossuportada apresentaram resultados bem inferiores, ficando com média de 6.63 pontos para a prótese superior, assim classificados como satisfeitos dentro da EVA, e 4.82 para a prótese inferior, tendo classificação como insatisfeitos (Tabela 2).

Tabela 2 - Média e desvio-padrão da satisfação do paciente quanto ao uso da prótese. Macapá, AP, Brasil, 2018.

\begin{tabular}{lccccc}
\hline Satisfação EVA & \multicolumn{2}{c}{ Prótese implantossuportada } & \multicolumn{2}{c}{ Prótese mucossuportada } & $\boldsymbol{p}(\boldsymbol{U})^{\star}$ \\
\cline { 2 - 6 } & Média & DP & Média & DP & \\
Prótese Superior & 9,39 & 0,50 & 6,63 & 0,71 & 0,01 \\
Prótese Inferior & 9,47 & 0,48 & 4,82 & 1,11 & \\
\hline
\end{tabular}

${ }^{*}$ Nível de significância $<0,05$ pelo teste U.

Fonte: Dados obtidos pelo autor no programa SPPS STATISTICS 22.

Uma das hipóteses para essa diferença significativa seria o fato de que as próteses implantossuportadas, por serem fixas e apresentarem inegavelmente maior retenção e estabilidade, oferecem ao usuário uma maior eficiência mastigatória, confiança e restabelecimento das relações interpessoais, causando bem-estar e, por consequência, melhor satisfação (AWAD MA, et al., 2004; PRECIADO A, et al., 2013).

Mesmo aparentando uma obviedade o fato de as próteses implantossuportada serem mais bem avaliadas e apresentarem usuários muito mais satisfeitos, existem alguns estudos, na literatura, que não mostram essa grande diferença de satisfação entre prótese mucossuportada e implantossuportada. Allen PF e Mcmillan AS (1999); em seu estudo com 93 pacientes, 48 usuários de prótese implantosssuportada e 35 usuários de prótese mucossuportada relataram que houve insatisfação, nos dois grupos, não havendo diferença estatística significativa nesta insatisfação.

Montero J et al. (2012); destacaram o Oral Health Impact Profile, para pacientes desdentados (OHIP-20sp), na população espanhola, e analisaram o fatorial do bem-estar e satisfação com a prótese. Não houve diferença estatística significativa entre usuários de prótese fixa e usuários de prótese removível.

Mesmo com uma quantidade razoável de artigos científicos mostrando que não há diferença significativa de satisfação com as próteses entre usuários de prótese total mucossuportada e usuários de prótese total implantossuportada, esses estudos são minoritários. A grande maioria da literatura médica vigente corrobora com o resultado, obtido neste estudo, de que usuários de prótese implantossuportada tendem a ter maior satisfação com sua prótese (MEIJER HJ, et al., 2003; THOMASON JM, et al., 2003; HARRIS D, et al., 2013).

Nas Tabelas 3 e 4 são apresentados os resultados de QV -OHIP -14Br. Nesta tabela observa-se o impacto da saúde bucal na Q.V por meio do questionário OHIP-14br. A população deste estudo que utiliza a prótese implantossuportada foi classificada com uma $Q V$ boa, $100 \%(n=48)$, enquanto a população que utiliza prótese mucossuportada, $73 \%(n=220)$, apresentou uma QV classificada como razoável, $22 \%(n=65)$ obtiveram uma classificação de má QV e apenas 5\% ( $n=14)$ estavam com uma QV classificada como boa (Tabela 3).

Tabela 3 - Classificação da qualidade de vida de pacientes que utilizam a prótese sobre implantes por meio do questionário OHIP- Macapá, AP, Brasil, 2018.

\begin{tabular}{lcccc}
\hline \multicolumn{1}{c}{ Classificação } & \multicolumn{2}{c}{ Prótese implantossuportada } & \multicolumn{2}{c}{ Prótese mucossuportada } \\
\cline { 2 - 5 } & $\mathrm{N}$ & $\%$ & $\mathrm{n}$ & $\%$ \\
\hline Boa qualidade de vida & 48 & 100 & 14 & 5 \\
Razoável qualidade de vida & 0 & 0 & 220 & 73 \\
Má qualidade de vida & 0 & 0 & 65 & 22 \\
\hline
\end{tabular}

*Fonte: Dados obtidos no SPPS STATISTICS 22.

Conforme a Tabela 4, a média da QV de pacientes que utilizam prótese implantossuportada foi de 3,24 $( \pm 01,32)$, menor do que os pacientes que utilizam prótese mucossuportada, média de $15,63( \pm 3,23)$. 
Tabela 4 - Média e desvio-padrão da Qualidade de Vida de pacientes que utilizam prótese. Macapá, AP, Brasil, 2018

\begin{tabular}{llll}
\hline Avaliação da QV & Média & DP & $\boldsymbol{p}(U)$ \\
Prótese Implantossuportada & 3,24 & 1,32 & $<0,0001$ \\
Prótese Mucossuportada & 15,63 & 3,23 & \\
\hline
\end{tabular}

*Nível de significância $<0,05$ pelo teste U.

${ }^{*}$ Fonte: Dados obtidos no SPPS STATISTICS 22.

Assim, na comparação das médias, percebe-se que aqueles indivíduos que fazem uso de prótese implantossuportada possuem uma melhor Q.V comparados aos que utilizam prótese mucossuportada, com $p<0,0001$ significativo. O OHIP é um instrumento específico para a mensuração do impacto da saúde bucal na qualidade de vida. Este questionário possui uma versão brasileira resumida chamada $\mathrm{OHIP} 14 \mathrm{Br}$, elaborada por Jacobovitz F (2003); e foi utilizado neste estudo. São inúmeros os estudos na literatura que testificam a qualidade, eficiência e exatidão deste instrumento, para verificar o impacto da saúde bucal na Q.V dos indivíduos (BRENNAN DF e SPENCER AJ, 2004).

Embora o OHIP 14 e suas variações locais (geralmente versões traduzidas do original e validadas em um determinado país) sejam instrumentos originalmente feitos, para mensurar o impacto da saúde bucal, de maneira geral, na Q.V, essa mensuração pode ser feita de maneiras mais específica ainda. Este estudo utilizou uma destas especificidades e aferiu o impacto da saúde bucal na Q.V apenas utilizando como critério o uso das próteses dentárias totais de indivíduos totalmente edêntulos.

Tal metodologia já foi bastante relatada na literatura, como é o caso do estudo de Zani SR, et al. (2009); Preciado A, et al. (2013); Kuo HC, et al. (2011), Heydecke G, et al. (2005); Perea C, et al. (2013); Furuyama C, et al. (2012); Kelly SAM, et al. (2012); Harris D, et al. (2013). Como o OHIP14 Br já foi validado na literatura, para sua utilização, em países de língua portuguesa, mais especificamente no Brasil (JACOBOVITZ F, 2003), e como é vasto o emprego deste instrumento para aferir o impacto da saúde bucal na qualidade de vida, usando apenas as próteses como referência de pesquisa, este autor não achou necessário validar este questionário para utilizar na população Amapaense, que é brasileira e fala português.

Muito embora possa parecer bastante óbvio que usuários de prótese total implantossuportada tenham Q.V superior, quando comparados a usuários de prótese total mucossuportada, pois o fator de retenção e estabilidade das próteses implantossuportada são inegavelmente superiores, ainda não havia nenhum estudo na literatura amapaense que provasse isso. Além do mais, apenas saber que um grupo tem Q.V melhor que o outro não é o suficiente, é necessário mensurar essa diferença e realmente estabelecê-la, em critérios da escala OHIP, quem tinha boa, razoável ou má qualidade de vida relacionada à saúde bucal.

Existem vários estudos na literatura médica vigente que mostram não haver diferença significativa na Q.V entre os dois grupos e outros que mostram até resultados antagônicos a este.

Allen AF e Mcmillan AS (1999); relatam um estudo clínico com 93 pacientes usuários de prótese total implantossuportada e mucossuportada e, ao final da pesquisa, ambos os grupos estavam insatisfeitos com as próteses, e o grupo de usuário de prótese total implantossuportada apresentou pior Q.V que o grupo de usuários de prótese total mucossuportada. Montero J et al. (2012); ao validarem o OHIP 20, também, compararam a Q.V de usuários de prótese total fixa e prótese total removível. O estudo mostrou não haver diferença significativa na Q.V entre os dois grupos.

Assim como os estudos citados acima, ainda, existe uma gama muito grande de outros estudos com resultados similares aos supracitados na literatura, entretanto, mesmo que esses estudos sejam numerosos, são minoritários. A grande maioria dos estudos mostram que usuários de prótese implantossuportada tendem a ter melhor Q.V, (HEYDECKE G, et al., 2005; FURUYAMA C, et al., 2012; VAN DER BILT A, et al., 2006; KELLY SAM, et al., 2012; HARRIS D, et al., 2013) e muitos revelam uma diferença substancial na Q.V, corroborando com os resultados obtidos neste estudo (ALLEN PF e MCMILLAN AS, 2003; PEREA C, et al., 2013; RAES S, et al., 2017; ATT W e STAPPERT C, 2003). 


\section{CONCLUSÃO}

Usuários de prótese total implantossuportada apresentaram satisfação com as próteses e Q.V relacionada à saúde bucal significativamente superior aos usuários de próteses totais mucossuportadas. $\mathrm{O}$ fato de a fixação das próteses totais implantossuportadas trazerem maior estabilidade, retenção e, consequentemente, melhor função mastigatória aos usuários, causa um aumento exponencial na qualidade de vida e satisfação com as próteses.

\section{REFERENCIAS}

1. ALLEN PF, MCMILLAN AS. A longitudinal study of quality of life outcomes in older adults requesting implant prostheses and complete removable dentures. Clinical Oral Implants Research, 2003; 14(2): 173-179.

2. ALLEN PF, MCMILLAN AS. The impact of tooth loss in a denture wearing population: an assessment using the Oral Health Impact Profile. Community Dental Health, 1999; 16(3): 176-180.

3. ATT W, STAPPERT C. Implant therapy to improve quality of life. Quintessence Int, 2003; 34(8): 573-581

4. AWAD MA, LUND JP, SHAPIRO SH et al. Oral health status and treatment satisfaction with mandibular implant overdentures and conventional dentures: a randomized clinical trial in a senior population. The International Journal of Prosthodontics, 2003; 16(4).

5. AWAD, MA, LUND JP, DUFRESNE E et al. Comparing the efficacy of mandibular implant-retained overdentures and conventional dentures among middle-aged edentulous patients: satisfaction and functional assessment. International Journal of Prosthodontics, 2003; 16(2).

6. BRENNAN DS, SPENCER AJ. Dimensions of oral health related quality of life measured by EQ-5D+ and OHIP-14. Health and quality of life outcomes, 2004; 2(1): 35.

7. FURUYAMA C, TAKABA M, INUKAI M et al. Oral health-related quality of life in patients treated by implant-supported fixed dentures and removable partial dentures. Clinical oral implants research, 2012; 23(8): 958-962.

8. HARRIS D, HÖFER S, O'BOYLE CA et al. A comparison of implant-retained mandibular overdentures and conventional dentures on quality of life in edentulous patients: a randomized, prospective, within-subject controlled clinical trial. Clinical oral implants research, 2013; 24(1): 96-103.

9. HEYDECKE G, LOCKER D, AWAD MA et al. Oral and general health-related quality of life with conventional and implant dentures. Community dentistry and oral epidemiology, 2003; 31(3): 161-168.

10. JACOBOVITZ F. Estudo sobre as qualidades psicométricas do Oral Health Impact Profile - 14. Tese (Doutorado em odontologia) - Faculdade de Odontologia de Ribeirão Preto da Universidade de São Paulo, 2003.

11. KELLY SAM, HYLAND RM, ELLIS JS et al. Development of a patient-based questionnaire about emotional and social issues related to eating with dentures. Journal of Dentistry, 2012; 40(8): 678-685.

12. KUO HC, CHEN JH, WU JH et al. Application of the oral health impact profile (OHIP) among Taiwanese elderly. Quality of Life Research, 2011; 20(10): 1707-1713.

13. MEIJER HJ, RAGHOEBAR GM, VAN'T HOF MA. Comparison of implant-retained mandibular overdentures and conventional complete dentures: a 10-year prospective study of clinical aspects and patient satisfaction. International Journal of Oral \& Maxillofacial Implants, 2003; 18(6): 879-885.

14. MONTERO J, CASTILLO-OYAGÜE R, LYNCH CD et al. Self-perceived changes in oral health-related quality of life after receiving different types of conventional prosthetic treatments: a cohort follow-up study. Journal of dentistry, 2013; 41(6): 493-503.

15. NICO LS. Saúde bucal autorreferida da população adulta brasileira: resultados da Pesquisa Nacional de Saúde 2013. Ciênc. Saúde Coletiva, 2016; 21(2): 389-398.

16. OLIVEIRA TRC, BORGES TF, MENDES FA et al. Risk of malnutrition in completely edentulous individuals (chapter XIV) in Malnutrition in the $21^{\text {st }}$ Century United States. Nova Science Publishers, 2007; 265(280): 265-280.

17. PARAGUASSU ÉC, CARDENAS AMC. Sociodemographic characterization of users of total tissue-supported and implantsupported prostheses in the municipality of macapá, Brazil. International Journal of Development Research, 2019; 9(02): 2608126084.

18. PEREA C, SUÁREZ-GARCÍA M J, DEL RÍO J et al. Oral health-related quality of life in complete denture wearers depending on their socio-demographic background, prosthetic-related factors and clinical condition. Medicina oral, patologia oral y cirugia bucal, 2013; 8(3): 371.

19. PRADO MMS. Avaliação da função mastigatória de pacientes reabilitados por próteses totais muco-suportadas. Dissertação (Mestrado em Reabilitação Oral) - Faculdade de Odontologia, Universidade Federal de Uberlândia, Uberlândia, 2004.

20. PRECIADO A, DEL RÍO J, LYNCH CD et al. Impact of various screwed implant prostheses on oral health-related quality of life as measured with the QoLIP-10 and OHIP-14 scales: A cross-sectional study. Journal of dentistry, 2013; 41(12): 1196-1207.

21. RAES S, RAES F, COOPER L et al. Oral health-related quality of life changes after placement of immediately loaded single implants in healed alveolar ridges or extraction sockets: a 5-year prospective follow-up study. Clinical oral implants research, 2017; 28(6): $662-667$.

22. SHEIHAM A, STEELE JG, MARCENES W et al. The relationship among dental status, nutrient intake, and nutritional status in older people. J Dent Res, 2001; 80(2): 408-413.

23. THOMASON JM, LUND JP, CHEHADE A et al. Patient satisfaction with mandibular implant overdentures and conventional dentures 6 months after delivery. Int J Prosthodont, 2003; 16(5): 467-473.

24. VAN DER BILT A, VAN KAMPEN FM, CUNE MS. Masticatory function with mandibular implant-supported overdentures fitted with different attachment types. Eur J Oral Sci, 2006; 14(3): 191-196.

25. ZANI SR, RIVALDO EG, FRASCA LC et al. Oral health impact profile and prosthetic condition in edentulous patients rehabilitated with implant-supported overdentures and fixed prostheses. Journal of Oral Science, 2009 ; 51(4): 535-543. 